\title{
Taiwankadsurins A, B and C, Three New C19 Homolignans from Kadsura philippinensis
}

\author{
Ya-Ching Shen, ${ }^{*}{ }^{\dagger}$ Yu-Chi Lin, ${ }^{\dagger}$ Yuan-Bin Cheng, ${ }^{\dagger}$ Yao-Haur Kuo ${ }^{\ddagger}$ \\ and Chia-Ching Liaw ${ }^{\dagger}$ \\ ${ }^{\dagger}$ Department of Marine Resources, National Sun Yat-Sen University, \\ Kaohsiung 804, Taiwan, Republic of China \\ ${ }^{\ddagger}$ National Research Institute of Chinese Medicine, Taipei, Taiwan, Republic of China
}

\section{List of Supporting Information}

1. ${ }^{1} \mathrm{H}$ NMR and ${ }^{13} \mathrm{C}$ NMR spectra of Taiwankadsurin $A$

2. ${ }^{1} \mathrm{H}$ NMR and ${ }^{13} \mathrm{C}$ NMR spectra of Taiwankadsurin $B$

3. ${ }^{1} \mathrm{H}$ NMR and ${ }^{13} \mathrm{C}$ NMR spectra of Taiwankadsurin $\mathrm{C}$ 
ᄂ2988 0

โ66ع0 ! -

$08290^{\circ}$

$\checkmark 9692^{\circ}$.

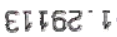

GLIVE'

980to

ob9bs'

टEट69. I

L90Iz

टLEEE'

$\checkmark \vdash$ GGE' 2

6699E' 2

OZ8LE'

$\angle 888^{\circ} \mathrm{C}-$

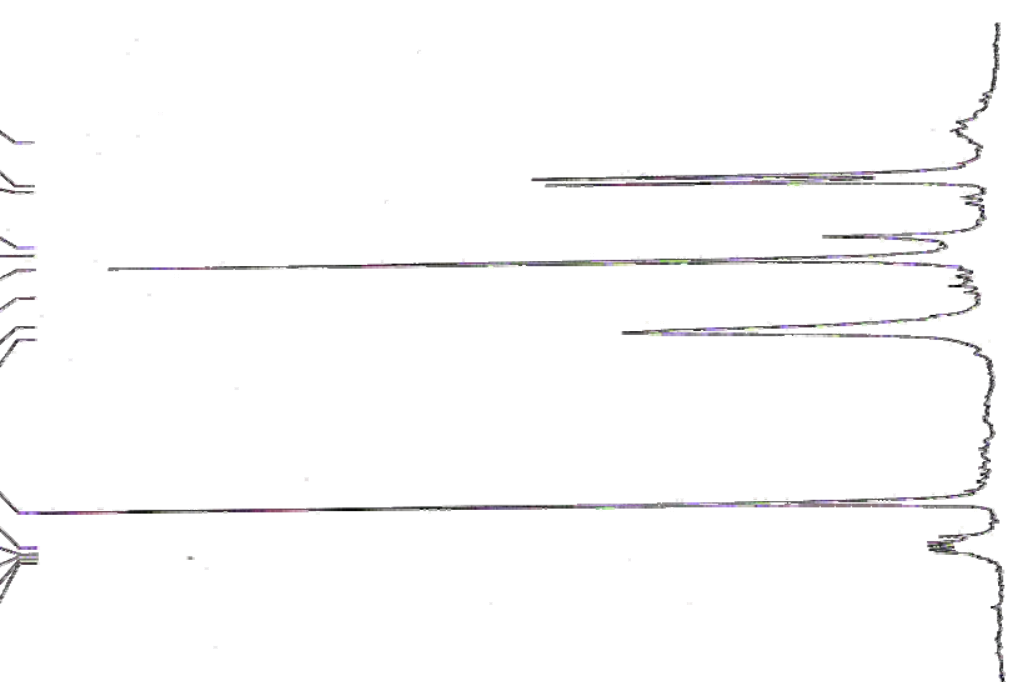

$=1822^{\circ} \varepsilon$

$=\square C E \nabla^{\circ}$
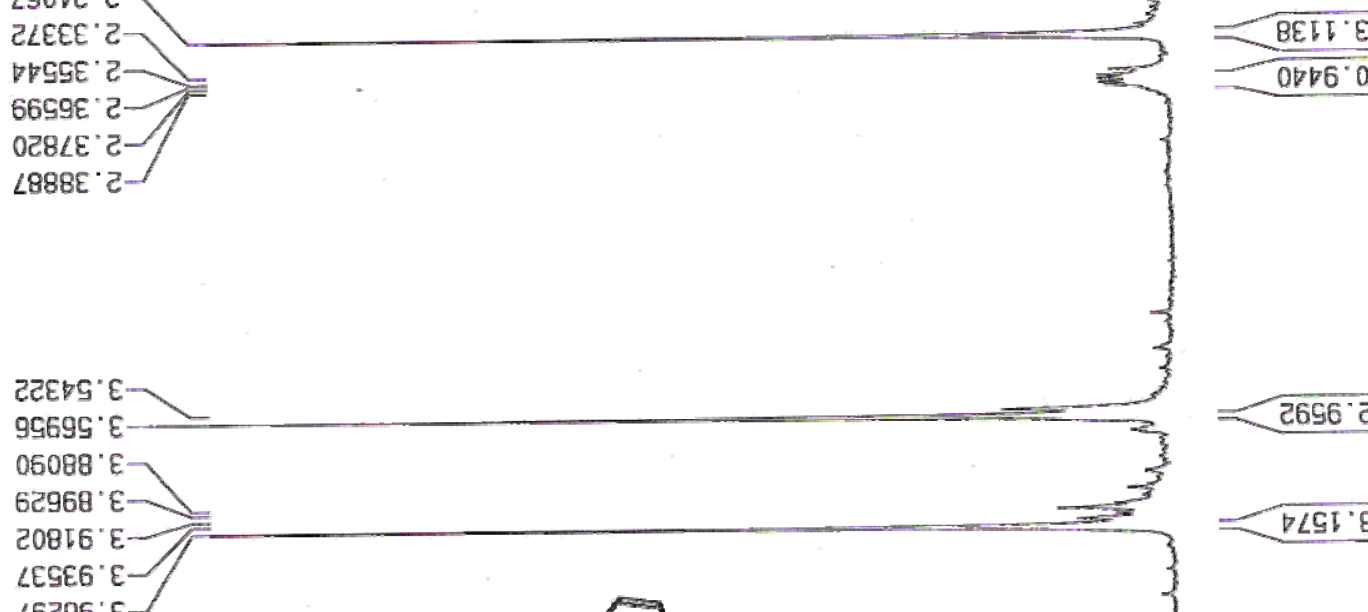

L6ट96 ' $E$ -

$88829^{\circ}$ LOE9G' $\square-$

E6986 $\circ$ $06610^{\circ} \mathrm{G}$

$29066^{\circ} \mathrm{S}$

15666' 9 $0 \angle D 20^{\circ} 9$ $\triangle E B Z 0^{\circ} 9$

8L280 9

$\checkmark 8160.9$

LI68' ' 9

$00865 \cdot 9$

टटॅ 09 ' 9

SL689' 9

$65669^{\circ} 9$

O2992' $\angle \longrightarrow$

$\angle G^{\prime} O G^{\prime} L$

टद6टद " $L$

OrGG9 $\angle$

टा8ट9 '

6tट59: $L$

G666ᄅ ' 8

GLDEE 8

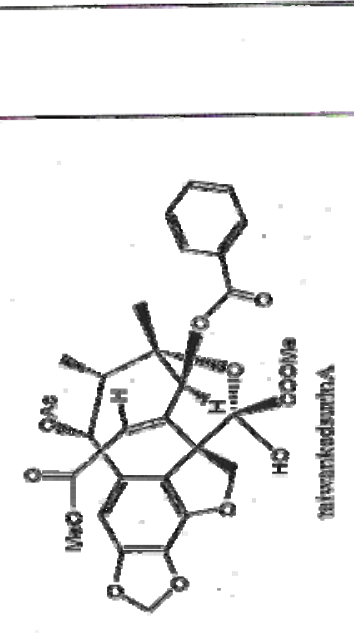

$= 2 \longdiv { 2 6 9 6 ^ { \circ } 2 }$

$=\nabla \angle G I^{\circ} \varepsilon$

$-\nabla$

16960

$0000^{\circ} \mathrm{I}$

$-1 n$

$$
-
$$

.

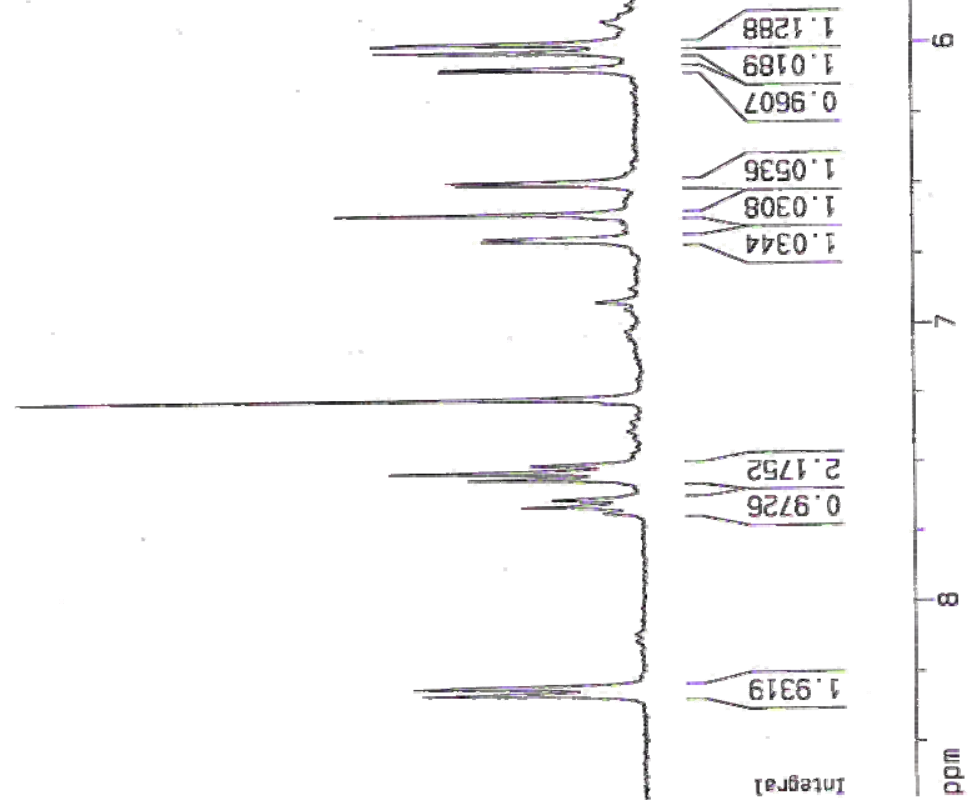


S3

$S \angle \angle * 8$

$\varepsilon 9 z^{-} \tau z$

$\angle \varepsilon \varepsilon^{*} \mathrm{Bz}$

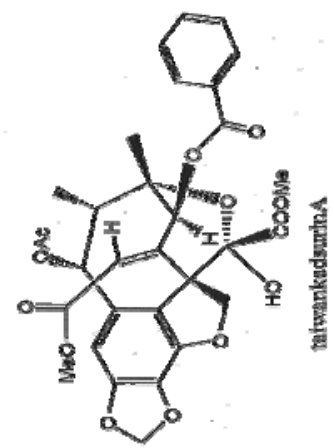

TSO $\angle S$ $\angle \angle Z^{*} S D$

$26 \angle-I S$

OTL* $E S$

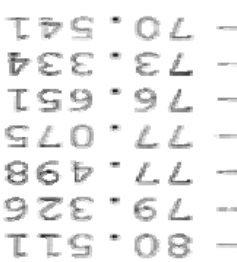

$16 s^{\circ} \angle 6$

590.66

$\varepsilon \varepsilon \sigma^{-\tau O} \tau$

$\varepsilon T \angle \cdot O T T$

$288^{\circ} \mathrm{TT}$

TEE・LIT

TOZ. $8 \mathrm{~T} T$

$2 T 8 \cdot \angle 2 T$

$\varepsilon T \pi \cdot 8 \tau T$

$\angle 88^{-82 T}$

$\varepsilon 09^{\circ} 0 \varepsilon \tau$

$\tau \tau I \cdot \nabla \varepsilon \tau$

$S \varepsilon \varepsilon-O S I$

SẼ * 0 I

$087^{-59 \tau}$

t t $\cdot 69$ t

$9 \angle O^{\circ} \mathrm{I} L \mathrm{I}$
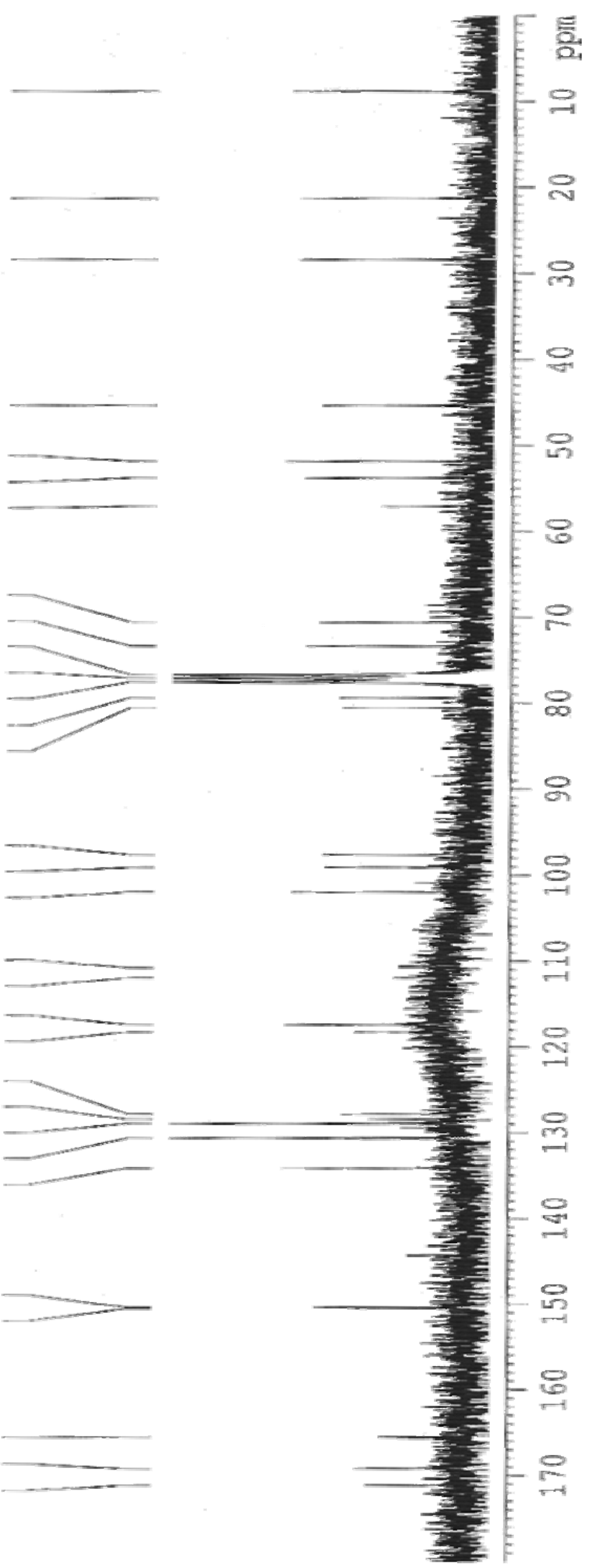


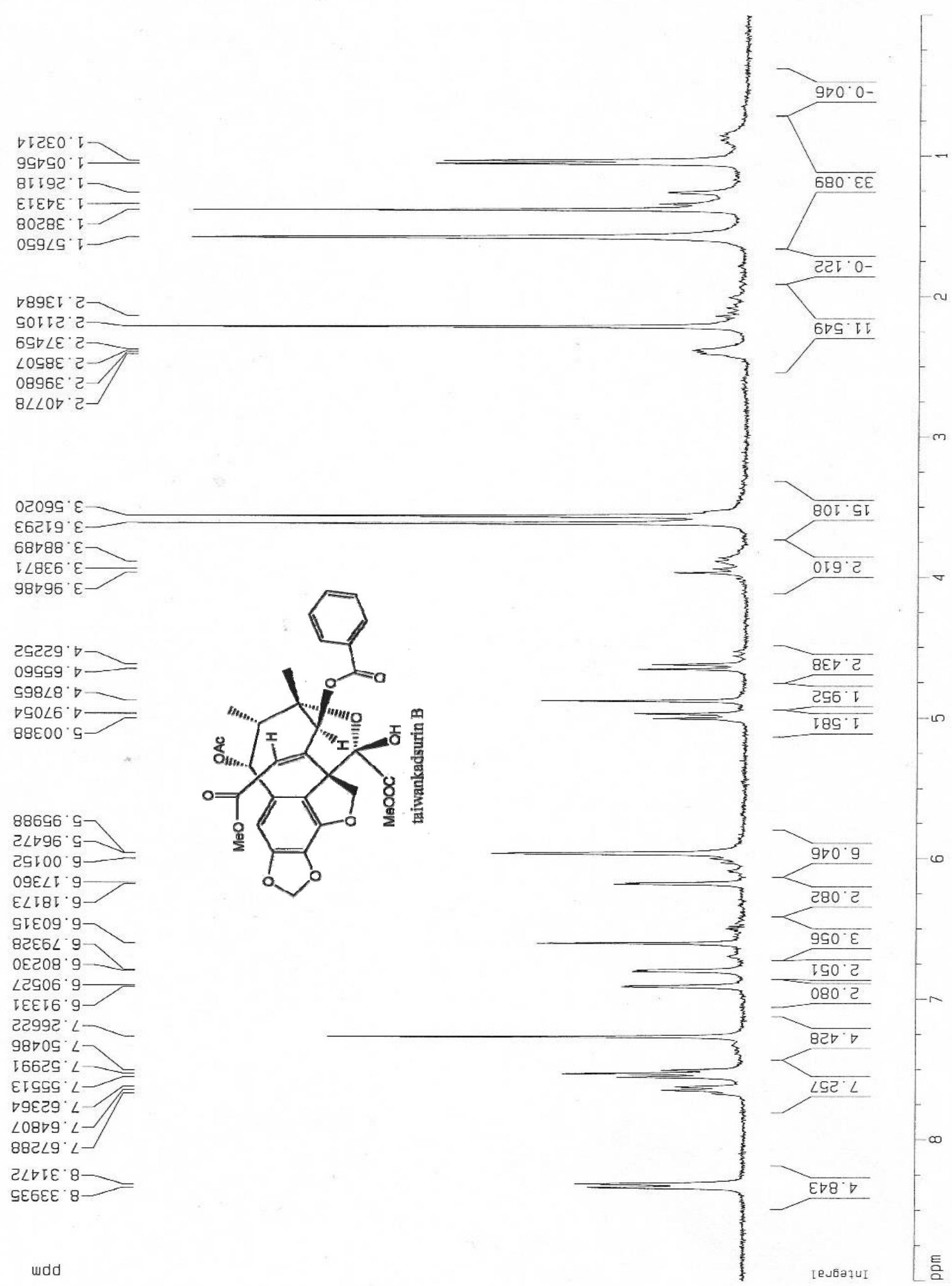


S5

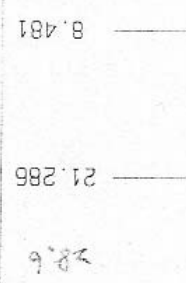

$280^{\circ} \mathrm{LL}$

$909^{\circ} L L$

$629.8 \angle$

$\nabla 28^{\circ} \angle 6-$

S8E 66
$\angle L 9 \cdot 101-$ $\angle 09^{\circ} 505$

$\nabla 60$ GI 9IS SII I $8 G^{\circ} 8 \mathrm{~T}$

I9LOCI-

$\angle 08^{\circ} \angle Z$

5ट8. 85

96S OET-

9७6 $E E I-$

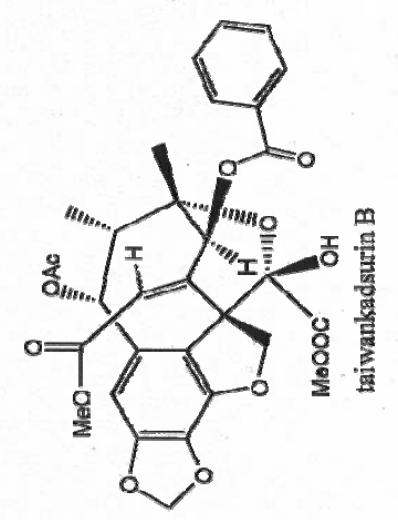

टจ9 $6 \nabla !$

bेt?

99999

E6!

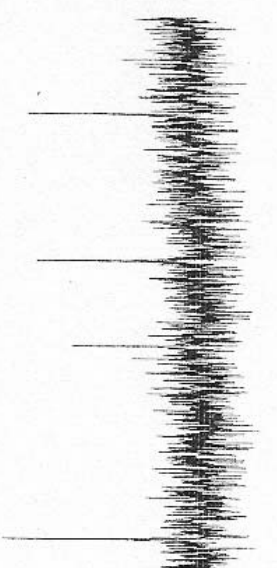




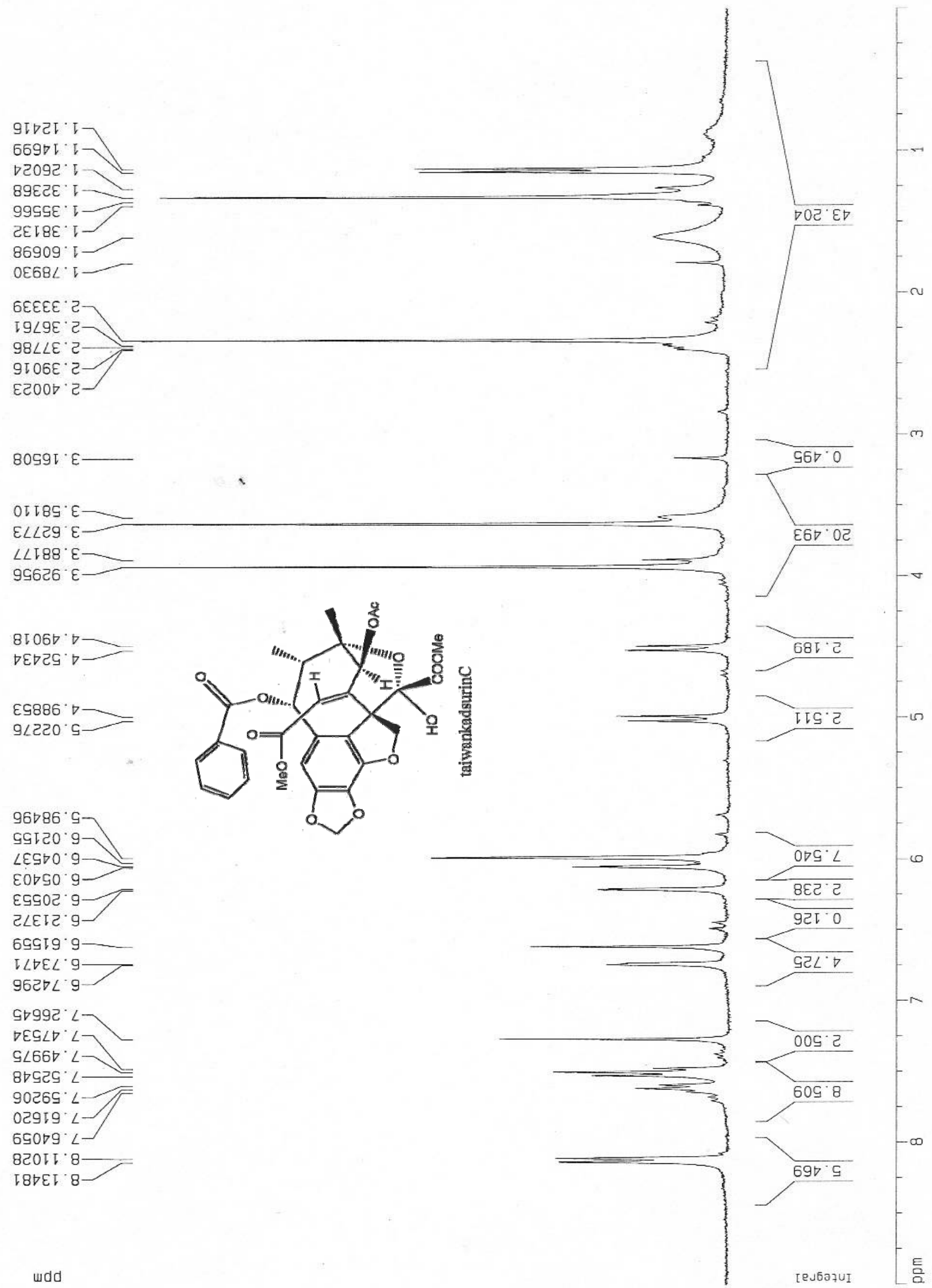


$\angle 90^{\circ} 6$

$\nabla 28 \cdot 02-$

$8 \varepsilon V^{\prime} 82$

$\varepsilon \forall \varepsilon \cdot G \forall$

$\nabla \angle 8^{\circ}$ IS

จ69' $89-$

$\neg 88^{\circ} 99$

69I' $T L$

OIT'EL

I $99 \cdot 9 L$

$\varepsilon 80^{\circ} L L$

$\angle O 9^{\prime} \angle L$

290 $6 \mathrm{~L}$

OBg' 08

$\mathrm{SGS}^{\prime} \angle 6$

9ह6. 86

$\checkmark 26^{\circ}$ IOI

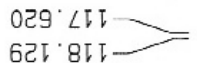

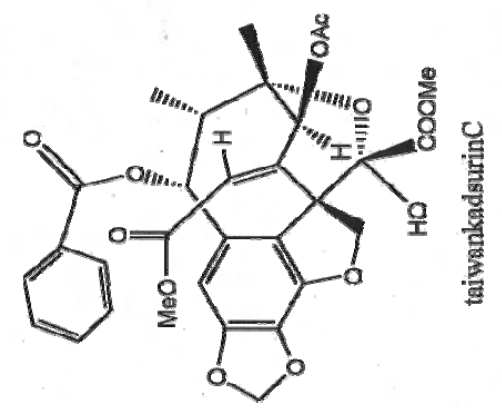

$688^{\circ} \angle 2 \downarrow$

$889^{\circ} 82 \mathrm{~T}$

$889^{\circ} 62 \mathrm{~T}$

$\triangle G I 0 E \downarrow$

टSE' EEI

$O \angle E^{\circ} O S I-$

9Gt. OGI $>$

$\nabla C L \angle 9 L$

ट6०. $991=$

$686^{\circ} 69 \mathrm{I}$

टEO. $\angle \mathrm{T}-\mathrm{C}$

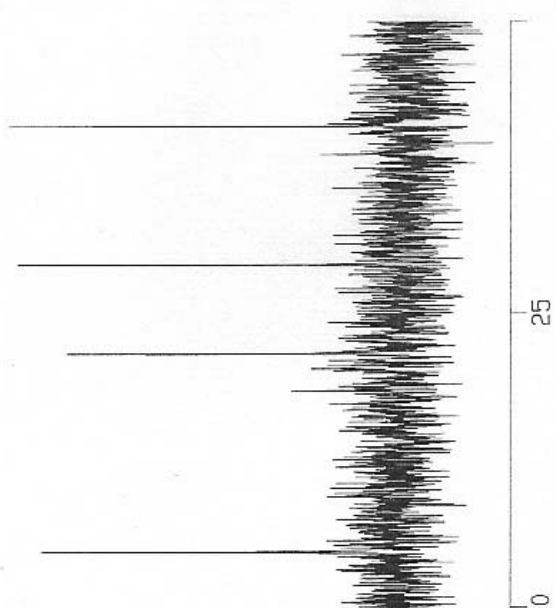

